\title{
Impact of Histogram Subset Selection on Classification using Multi-scale LBP-Operators
}

\author{
Sebastian Hegenbart ${ }^{1}$, Andreas Uhl ${ }^{1}$, Andreas Vécsei ${ }^{2}$ \\ ${ }^{1}$ Department of Computer Sciences, University of Salzburg \\ ${ }^{2}$ St. Anna Children's Hospital, Vienna \\ shegen@cosy.sbg.ac.at
}

\begin{abstract}
Multi-scale Local Binary Pattern based operators are used to extract features from duodenal texture patches with histological ground truth in case of pediatric celiac disease. The multi-scale LBP combined with color channels and possibly other filters lead to a high number of computed histograms. The impact of histogram subset selection on the overall classification rates using two feature subset selection algorithms (SFS and SBS) with three LBP-based operators is analyzed and the applicability of these techniques validated.
\end{abstract}

\section{Introduction}

Celiac disease is a complex autoimmune disorder in genetically predisposed individuals of all age groups after introduction of gluten containing food. During the course of the disease, hyperplasia of the enteric crypts occurs and the mucosa eventually looses its absorptive villi thus leading to a diminished ability to absorb nutrients. People with untreated celiac disease are at risk for developing various complications like osteoporosis, infertility and other autoimmune diseases including type 1 diabetes. Endoscopy with biopsy is currently considered the gold standard for the diagnosis of celiac disease. During endoscopy at least four duodenal biopsies are taken. Microscopic changes within these specimen are classified by a histological analysis according to a classification scheme by Oberhuber et al. [1]. The benefits of an automated support tool for diagnosis are many. Among them are an improved reliability of diagnosis, supported targeting of biopsies and more efficient use of time and manpower.

The Local Binary Pattern (LBP) operator is invariant to monotonic intensity variations which is beneficial to texture classification in environments with varying lighting conditions. This property makes the method interesting for classifying endoscopic images. In the context of LBP many modifications and related operators have been suggested over the years. A prominent modification that is often neglected across the literature is the multi-scale approach suggested by Mäenpää [2]. This approach is based on low-pass filtering combined with appropriate filter sizes and operator radii to improve the operators' spatial support area. Using this extension, combined with color channels and possibly other filters, the number of computed histograms is considerably higher than the 
Table 1. Distribution of image data.

\begin{tabular}{lllllll}
\hline & \multicolumn{2}{l}{ Image-Set 1 } & \multicolumn{3}{c}{ Image-Set 2 } \\
& Class $_{0}$ & Class $_{1}$ & Total & Class $_{0}$ & Class $_{1}$ & Total \\
\hline Bulbus Duodeni & 153 & 120 & 273 & 187 & 70 & 257 \\
Pars Descendens & 132 & 164 & 296 & 115 & 58 & 173 \\
\hline
\end{tabular}

common approach of using a combination of two or three different parameterizations of the operator. It is unclear how a high number of histograms affects the classification. To study the effects we use two feature subset selection schemes to find optimal suitable combinations of histograms. We analyze the impact of the subset selection and validate the applicability of these techniques using two distinct image sets.

\section{Materials and Methods}

The image test set used, contains images taken during duodenoscopies at the St. Anna Children's Hospital using pediatric gastroscopes without magnification. Images were recorded by using the modified immersion technique, which has been shown to be beneficial to automated classification by Hegenbart et al. [3]. There are two duodenal regions with completely different geometric properties, i.e. the duodenal Bulb and the Pars Descendens. Accordingly, we chose to separate the images into two distinct sets. Texture patches with a fixed size of $128 \times 128$ pixels were extracted from the full sized frames, a size which turned out to be optimally suited in previous experiments [3]. The ground truth for the texture patches used in experimentation was determined by histological examination of biopsies from corresponding regions. In the following, we aim at a two class problem with the classes Class $_{0}$ as the class representing healthy tissue and Class ${ }_{1}$ representing texture patches showing villous atrophy. Table 1 shows the number of images available per considered class. For evaluation two distinct set of images for both duodenal regions denoted as Set-1 and Set-2 were assembled. This happened at two different points in time, the specific sets reflect the time intervals where the images were captured.

\section{$2.1 \quad$ Feature Extraction}

The basic LBP operator was introduced to the community by Ojala et al. [4]. We use three operators that are based on LBP to conduct our experiments. The operators are LTP (Local Ternary Patterns, [5]), ELBP (extended Local Binary Patterns, [6]), and the LBP operator combined with a contrast measure (LBPC, [4]). The entire family of operators is used to model a pixel neighborhood in terms of pixel intensity differences. The operators assign a binary label to each possible pixel neighborhood. The distributions of these labels are then used as features. The distributions are represented by histograms. We compute the 
pattern distributions for each color channel (RGB), each LBP-Scale (1-3) as well as filter orientation (in case of the extended LBP based operators: horizontal, vertical and diagonal). In total this is 9-histograms for LTP and LBPC, and 27histograms for ELBP. For each histogram, only a subset of dominant patterns known as the uniform patterns [7] which make up the majority of discriminative patterns is used. This subset consists of 58-patterns for 8 considered neighbors.

\subsection{Histogram Subset Selection}

Depending on the specific operator, at least 9 and at maximum 27 histograms are computed for a single image. A single LBP histogram can be interpreted as a macro feature. Therefore the terms histogram subset selection and feature subset selection share the same meaning. Feature subset selection techniques are usually applied for two reasons.

- Result Optimization: Probably not all parameters combinations are equally well suited for describing the specific textural properties. Even more, when computing a large number of histograms, this set could contain a few "bad" histograms which reduce the discriminative power.

- Reduction of Dimensionality: Depending on the chosen classification method large feature vectors might be suboptimal in terms of computational complexity and classification performance. Feature subset selection can be used to reduce the number of considered histograms and therefore the final feature vector dimensionality.

The applied algorithms were the Sequential Forward Selection algorithm (SFS, [8]) and the Sequential Backward Selection algorithm (SBS, [8]). Please note, that due to the imbalance of image number in the specific classes among the two image sets we chose the average classification rate of both classes as optimization criterion.

\subsection{Classification}

The k-nearest neighbors $(\mathrm{kNN})$ classifier was used for classification. A rather weak classifier was chosen to give more emphasis on the selected histogram combinations. After the histogram subset selection the candidate histograms were combined and treated as a single histogram. The classification is based on the histogram intersection distance between two histograms. The optimal k-value was found in a range from 1 to 25 .

\section{Results}

Tables 2 and 3 demonstrate the effect of using subset selection on the set of histograms. For each experiment the entire set of histograms was computed using the specific operator and both image sets (Set-1 and Set-2). The algorithms mentioned in section 2.2 were then used to select subsets for each image set. The sets 
Table 2. Classification results of images from the Bulbus-sets.

\begin{tabular}{cccccccccccc}
\hline \multicolumn{3}{c}{ Image Set-1 } & \multicolumn{10}{c}{ Image Set-2 } \\
& Class $_{0}$ & Class $_{1}$ & Total & $\Delta$ All & $\Delta$ Set1 & Class $_{0}$ & Class $_{1}$ & Total & $\Delta$ All & $\Delta$ Set2 \\
\hline SBS LBPC & 97.39 & 92.50 & 95.24 & -0.36 & -1.83 & 98.04 & 95.00 & 96.70 & +0.59 & -1.74 \\
ELBP & 94.12 & 91.67 & 93.04 & +0.37 & -2.56 & 98.93 & 94.29 & 97.67 & +0.79 & -0.77 \\
LTP & 98.69 & 93.33 & 96.34 & +0.37 & -0.36 & 98.93 & 84.29 & 94.94 & +0.39 & -1.56 \\
\hline SFS LBPC & 97.39 & 92.50 & 95.24 & -0.36 & -1.83 & 96.73 & 97.50 & 97.07 & +0.96 & -1.37 \\
ELBP & 94.77 & 84.17 & 90.11 & -2.56 & -6.59 & 98.40 & 95.71 & 97.67 & +0.79 & -0.77 \\
LTP & 96.73 & 95.83 & 96.34 & +0.37 & -1.40 & 98.93 & 90.00 & 96.50 & +1.95 & +1.56 \\
\hline
\end{tabular}

Table 3. Classification results of images from the Pars-sets.

\begin{tabular}{cccccccccccc}
\hline \multicolumn{3}{c}{ Image Set-1 $^{2}$} & \multicolumn{1}{c}{ Image Set-2 } \\
& Class $_{0}$ & Class & & Total & $\Delta$ All & $\Delta$ Set1 & Class $_{0}$ & Class $_{1}$ & Total & $\Delta$ All & $\Delta$ Set2 \\
\hline SBS LBPC & 66.67 & 95.12 & 82.43 & -1.35 & -3.72 & 79.55 & 91.46 & 86.15 & -6.34 & -6.91 \\
ELBP & 75.76 & 91.46 & 84.46 & +0.68 & -0.34 & 90.43 & 82.76 & 87.86 & -0.58 & -2.31 \\
LTP & 84.85 & 85.37 & 85.14 & -2.02 & -3.04 & 90.43 & 86.21 & 89.02 & +0.58 & -1.15 \\
\hline SFS LBPC & 62.88 & 92.68 & 79.39 & -4.39 & -6.76 & 79.55 & 91.46 & 86.15 & -6.34 & -6.91 \\
ELBP & 73.48 & 88.41 & 81.76 & -2.02 & -4.73 & 88.70 & 81.03 & 86.13 & -2.31 & -2.31 \\
LTP & 73.48 & 89.02 & 82.09 & -5.07 & -6.42 & 89.57 & 87.93 & 89.02 & +0.58 & -1.73 \\
\hline
\end{tabular}

were optimized until no new local maximum considering the classification rate could be found. The found subsets of Set-1 were then used to classify the images from Set-2 and vice versa. We compare the overall classification rates of these experiments with the rates gained by using the entire set of histograms without performing histogram subset selection (column $\Delta$ All) and the rates gained by optimizing the feature subset for the specific image set the classification is actually performed on (we expect this to be over fitted, column $\Delta$ Set1 or $\Delta$ Set2). We denote an increase in overall classification rate with a elqq + " and a decrease with a "-".

\section{Discussion}

We can see that using feature subset selection algorithms to find a reliable subset of histograms in case of multi-scale LBP is reasonable in case of the duodenal Bulb. The final feature vector dimensionality could be reduced and most classification rates be improved. The SBS method provides slightly more reliable results in terms of classification rates but SFS is more efficient in terms of feature vector dimensionality reduction. Comparing the results with the optimized results for the specific datasets, we see that the average loss in classification rate is approximately $1.86 \%$. This indicates that the optimized subsets are slightly over fitted. In contrast to the result of the Bulbus-experiments the results of the 
Pars-experiments show a general decrease in overall classification rate. Again the SBS method provided more reliable results as compared to SFS, however in general no reliable subsets of histograms could be found to guarantee stable classification rates. Compared to the Bulbus-experiments the histogram subsets are even more over fitted. The average loss in classification rate is over $3.86 \%$ in this case. The Pars-set contains two different types of images (and perspectives), namely the classical perspective perpendicular to the mucosa and the perspective into the direction of the center of the lumen (Fig. 1). Images exhibiting the latter perspective cannot be well described by LBP based operators.This leads to unreliable histograms and affects the subset selection. To overcome this limitation, a pre-classification of the images contained in the Pars-set, combined with a separate classification of each type of perspective could be introduced.

We see that histogram subset optimization can be a feasible option for both, reducing feature vector dimensionality and improving classification performance. By using distinct test- and training-sets over fitting can be avoided.
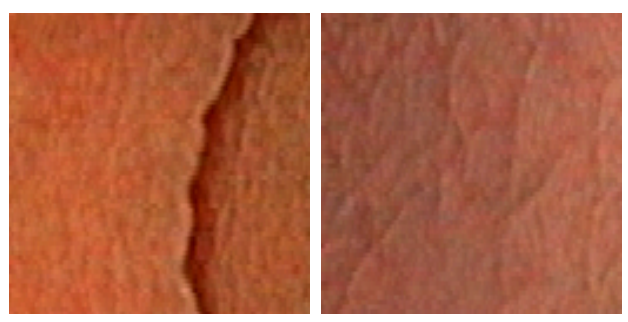

Fig. 1. Images from the Pars Descendens showing the two perspectives.

\section{References}

1. Oberhuber G, Granditsch G, Vogelsang H. The histopathology of coeliac disease: time for a standardized report scheme for pathologists. Eur J Gastroenterol Hepatol. 1999;11:1185-1194.

2. Mäenpää T. The Local Binary Pattern Approach to Texture Analysis: Extensions and Applications [PhD Thesis]. University of Oulu; 2003.

3. Hegenbart S, Kwitt R, Liedlgruber M, et al. Impact of duodenal image capturing techniques and duodenal regions on the performance of automated diagnosis of celiac disease. Proc ISPA. 2009; p. 718-723.

4. Ojala T, Pietikäinen M, Harwood D. A comparative study of texture measures with classification based on feature distributions. Pattern Recognit. 1996;29(1):51-59.

5. Tan X, Triggs B. Enhanced local texture feature sets for face recognition under difficult lighting conditions. Lect Notes Computer Sci. 2007;4778:168-182.

6. Huang X, Li S, Wang Y. Shape localization based on statistical method using extended local binary pattern. Proc ICIG. 2004; p. 1-4.

7. Mäenpää T, Ojala T, Pietikäinen M, et al. Robust texture classification by subsets of local binary patterns. Proc ICPR. 2000;3:3947.

8. Jain A, Zongker D. Feature selection: evaluation, application, and small sample performance. IEEE Trans Pattern Anal Mach Intell. 1997;19:153-158. 